Progress in Nuclear Science and Technology

Volume 6 (2019) pp. 73-76

\title{
ARTICLE
}

\section{Development of a wireless multisensor active personal dosimeter-tablet system}

\author{
Toshioh Fujibuchi $^{\mathrm{a}^{*}}$, Airi Inoue ${ }^{\mathrm{a}}$, Yang Ishigaki ${ }^{\mathrm{b}}$ and Yoshinori Matsumoto ${ }^{\mathrm{c}}$ \\ ${ }^{a}$ Kyushu University, 3-1-1 Maidashi, Higashi-ku, Fukuoka, Fukuoka, 812-8582, Japan; ${ }^{b}$ The University of Electro-Communications, \\ 1-5-1 Chofugaoka, Chofu-shi, Tokyo, 182-8585, Japan; ' Keio University, 3-14-1 Hiyoshi, Kouhoku-ku, Yokohama, Kanagawa, \\ 223-8522, Japan
}

\begin{abstract}
Medical staff who perform interventional radiology face the risk of exposure to relatively high doses of primary radiation and scattered radiation emitted from patients' bodies. For monitoring this exposure, we developed a wireless multisensor dosimeter that we named "Pocket Dose," which provides real-time visualization of dose levels on an Android tablet screen. In this study, we investigate the characteristics of the energy and dose rates of the developed system comprising silicon PIN photodiode detectors, a signal transmitter, and a tablet for data display. The detection unit is equipped with two types of sensors: one for measuring low dose rates and the other for measuring high dose rates. In addition, four detectors are connected to the transmitter with a one-meter-long cable. These detectors are designed to measure the unequal radiation exposures at the chest, neck, and fingers of medical staff. The signals from the detectors transmit count information to the tablet using Bluetooth. The dose-rate linearities of the high-sensitivity and low-sensitivity sensors are confirmed to be less than 14.8 and $82.8 \mathrm{mSv} / \mathrm{h}$, respectively. The system has great potential for energy correction using the energy spectra information. Using this system with wireless communication, real-time management of staff's exposure becomes easy. Moreover, high expandability is expected since it uses a tablet.
\end{abstract}

Keywords: monitoring system; Bluetooth wireless module; X-ray; silicon PIN photodiode sensor; active personal dosimeter

\section{Introduction}

Medical staff working in the field of interventional radiology (IVR) face the risk of exposure to relatively high doses of scattered radiation emitted from patients' bodies [1,2]. For example, during fluoroscopy in interventional cardiology, the dose at the location of the cardiologist corresponds to a scattered dose in the range from 1 to $14 \mathrm{mSv} / \mathrm{h}$ [3]. For occupational workers in planned exposure situations, the International Commission on Radiological Protection (ICRP) recently recommended an equivalent dose limit of $20 \mathrm{mSv} /$ year for the lens of the eye, averaged over a defined period of five years, with single-year exposures limited to $50 \mathrm{mSv}$ [4]. Revision of the "Act on Prevention of Radiation Hazards due to Radioisotopes, etc." based on this new exposure limit will increase the risk of cataract for IVR staff exceeding the annual dose limit. Therefore, management of additional occupational exposure is important $[5,6]$.

To manage occupational exposure, medical staff wear protective aprons and use passive-type personal dosimeters, such as Glass Badge or QuIxel Badge, for radiation-exposure monitoring. Passive-type personal

*Corresponding author. Email: fujibuch@hs.med.kyushu-u.ac.jp dosimeters are the most popular dosimeters among clinical practitioners for personal monitoring; however, they cannot measure exposure doses in real time. Individual monitoring in real time is helpful for determining exposure doses during IVR [7-10]. Furthermore, by displaying the dose wirelessly on the monitor, it is possible to easily determine the dose, avoiding the need for complicated procedures [11-13]. For exposure monitoring, we developed a multisensor wireless dosimeter system that we named "Pocket Dose," which provides real-time visualization of the dose levels on an Android tablet screen. In this study, we investigated the characteristics of the energy spectra and dose rate of this system.

\section{Materials and methods}

\subsection{Pocket dose}

Pocket Dose is a wireless multisensor active personal dosimeter-tablet system. It is equipped with four detectors, each comprising two types of silicon PIN photodiode sensors: S6755 (effective area: $26.4 \mathrm{~mm}^{2}$, Hamamatsu Photonics K.K, Japan) (hereinafter called "Sensor 1") for low dose rates, and S2506 (effective 
area: $7.67 \mathrm{~mm}^{2}$, Hamamatsu Photonics K.K, Japan) (hereinafter called "Sensor 2") for high dose rates. These detectors are connected to a wireless transmitter using a one-meter-long cable. The weights of the detector, cable, and transmitter are 16, 15, and $135 \mathrm{~g}$, respectively. The four detectors are designed to measure the unequal radiation exposures at the chest, neck, and wrists of medical staff. The signals from the detectors transmit count information to the tablet using Bluetooth 4.0. The tablet operates on Android OS, and a dedicated application for Pocket Dose is installed. A picture of Pocket Dose and its components is shown in Figure 1. A photograph of the detectors placed on the chest, neck, and wrist is shown in Figure 2. The transmitter can be carried in the pocket. This system allows nonuniform exposure management as one person can wear multiple detectors.

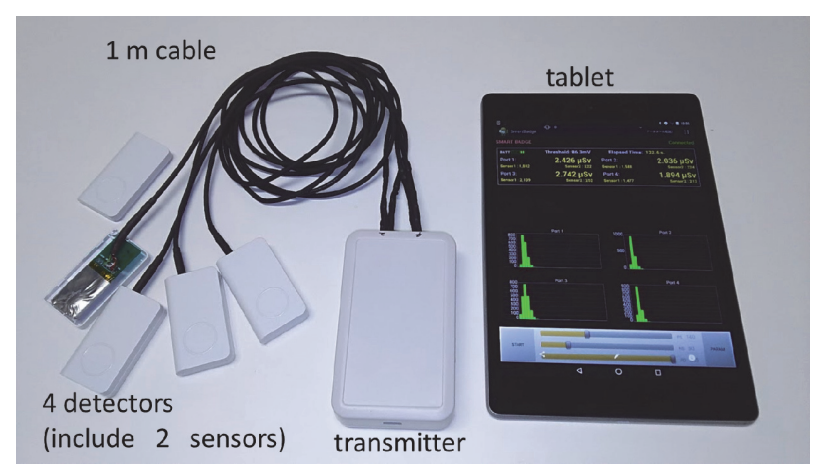

Figure 1. Picture of Pocket Dose.

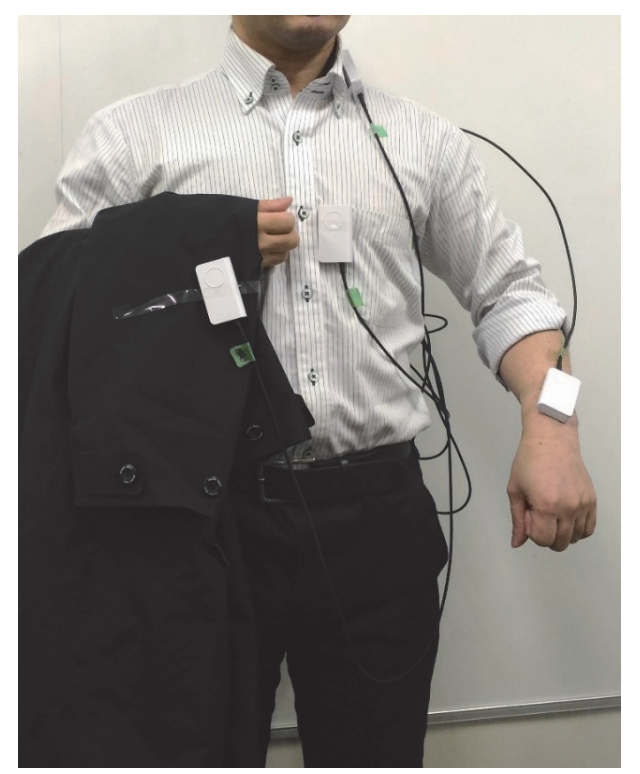

Figure 2. Photograph of the detectors placed on the chest, neck, and wrist for nonuniform exposure management.

The Pocket Dose application displays the following: the $1 \mathrm{~cm}$ dose equivalent rate converted according to the conversion coefficient set by the user, the detected counts of each sensor, and the spectra of 32 channels according to the pulse height measured by each detector.
The acquired data can be transferred via e-mail from the tablet. The pulse threshold value and the reverse-bias voltage of each PIN photodiode can be adjusted using the application. The application user interface is shown in Figure 3.

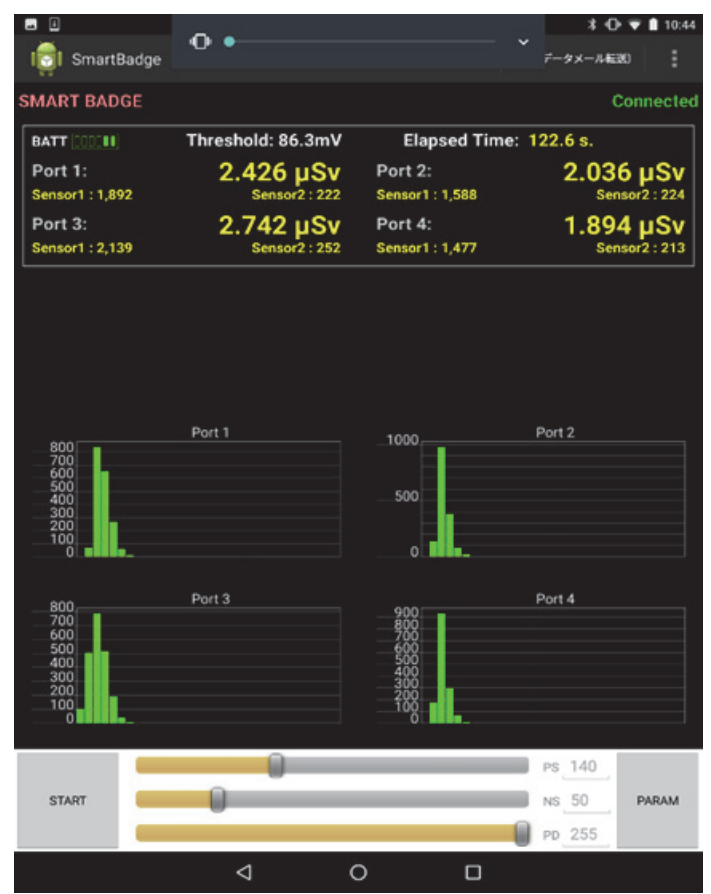

Figure 3. User interface of the Pocket Dose application.

\subsection{Estimation of energy spectra using Pocket Dose}

In this study, we used a radiography machine (UD 150 L-30: Shimadzu Corporation, Japan) as an X-ray source. An elliptical water phantom prepared as a scattered radiation source was used for simulating the patient. The focus-to-image detector distance was set as $100 \mathrm{~cm}$. A CdTe spectrometer (EMF123: EMF Japan, Japan) and Pocket Dose were placed on the side at a distance of $100 \mathrm{~cm}$ from the patient, and the energy spectrum was measured. The energy spectrum was measured by sensor 1 in the Pocket dose. The X-ray tube voltage were set at different values of $60,80,100$, and $120 \mathrm{kV}$. At each tube voltage, the beam current was adjusted so that the dose rate at the detector was 1 $\mathrm{mGy} / \mathrm{h}$. The spatial arrangement of the different components during the energy spectra measurement is shown in Figure 4.

\subsection{Estimation of reproducibility}

To estimate the reproducibility of Pocket Dose, we used a semiconductor detector (DDX6-WL: Radcal Corporation, USA) as the reference dosimeter. The setup used was the same as that described in Section 2.2 and Figure 4. The parameters used for irradiation in the reproducibility experiment are as follows: $80 \mathrm{kV}$ tube voltage, $100 \mathrm{~mA}$ tube current, and $0.1 \mathrm{~s}$ irradiation time. The measurement was repeated 10 times using both Sensor 1 and Sensor 2 of Detector 1 . 


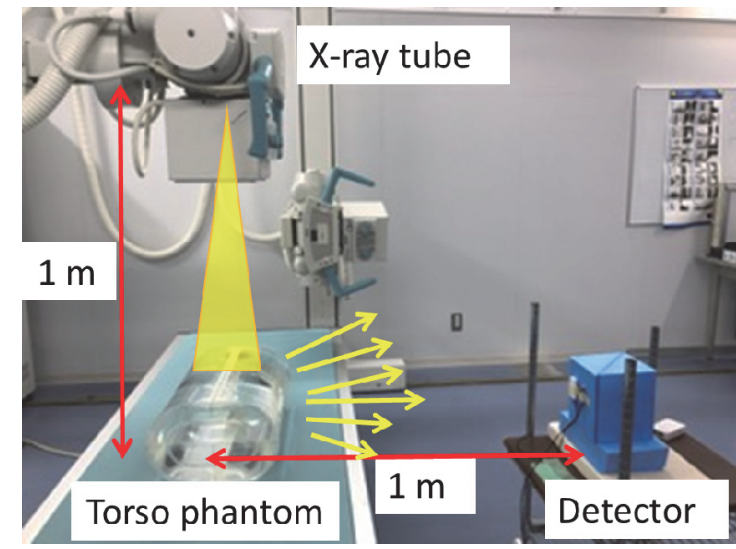

Figure 4. Setup for energy spectra measurement.

\subsection{Estimation of dose rate characteristics}

In the dose rate dependence experiment, Pocket Dose and DDX6-WL were simultaneously exposed to scattered X-rays from the torso phantom. We investigated the linearity between the air kerma rate and the count rate by changing the tube current from 25 to $400 \mathrm{~mA}$ and the distance between the phantom and the detector from 50 to $100 \mathrm{~cm}$ under radiographic conditions.

\section{Results and discussion}

\subsection{Estimation of energy spectra using Pocket Dose}

The pulse height distribution measured by the CdTe detector is shown in Figure 5, and that measured by Pocket Dose is shown in Figure 6. Both pulse height distributions show peaks in the low channel, and the count continued up to the high channel, depending on the tube voltage.

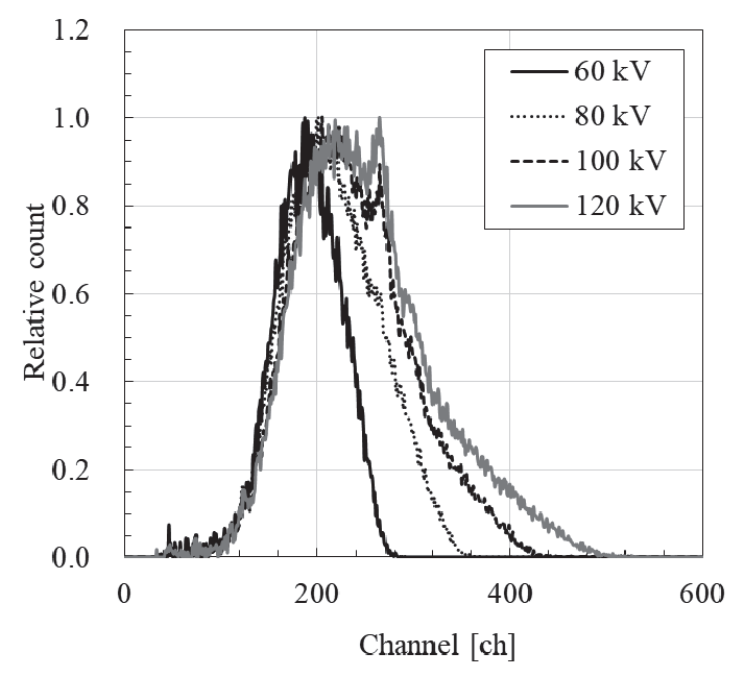

Figure 5. Pulse height distribution measured using the CdTe spectrometer.

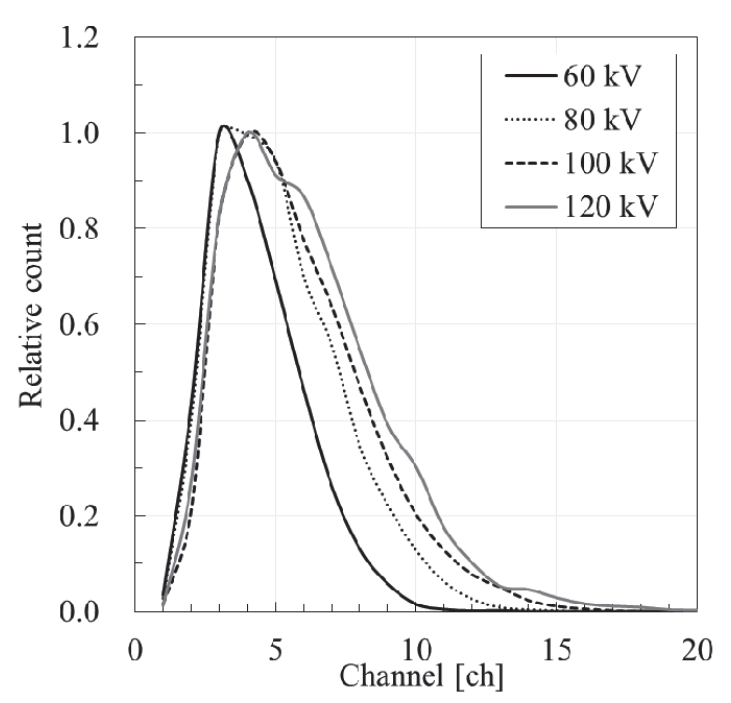

Figure 6. Pulse height distribution measured using Sensor 1 of Pocket Dose.

Based on these results, we infer that the energy characteristics of this dosimeter can be kept constant by adjusting the dose equivalent conversion coefficient for each channel.

\subsection{Estimation of reproducibility}

Table 1 shows the estimation of reproducibility. The coefficients of variation for Sensors 1 and 2 are $4.5 \%$ and $18.2 \%$, respectively.

Table 1. Estimation of reproducibility.

\begin{tabular}{|c|c|c|c|}
\hline \multicolumn{4}{|c|}{ Detector 1} \\
\hline & $\begin{array}{l}\text { Sensor } 1 \\
\text { [counts] }\end{array}$ & $\begin{array}{c}\text { Sensor } 2 \\
\text { [counts] }\end{array}$ & $\begin{array}{c}\text { DDX-WL } \\
{[\mu \mathrm{Gy}]}\end{array}$ \\
\hline Average & 243 & 33 & 81 \\
\hline $\begin{array}{l}\text { Standard } \\
\text { deviation }\end{array}$ & 11 & 6 & 2 \\
\hline $\begin{array}{l}\text { Coefficient of } \\
\text { variation }[\%]\end{array}$ & 4.5 & 18.2 & 2.5 \\
\hline
\end{tabular}

\subsection{Estimation of dose rate characteristics}

The count rate characteristics of Sensors 1 and 2 are shown in Figure 7. The low-dose-rate sensor (Sensor 1) showed a coefficient of determination of 0.94 up to 14.8 $\mathrm{mGy} / \mathrm{h}$ in a linear approximation. The high-dose-rate sensor (Sensor 2) showed a linearity approximation coefficient of determination of 0.90 up to $82.8 \mathrm{mGy} / \mathrm{h}$. 


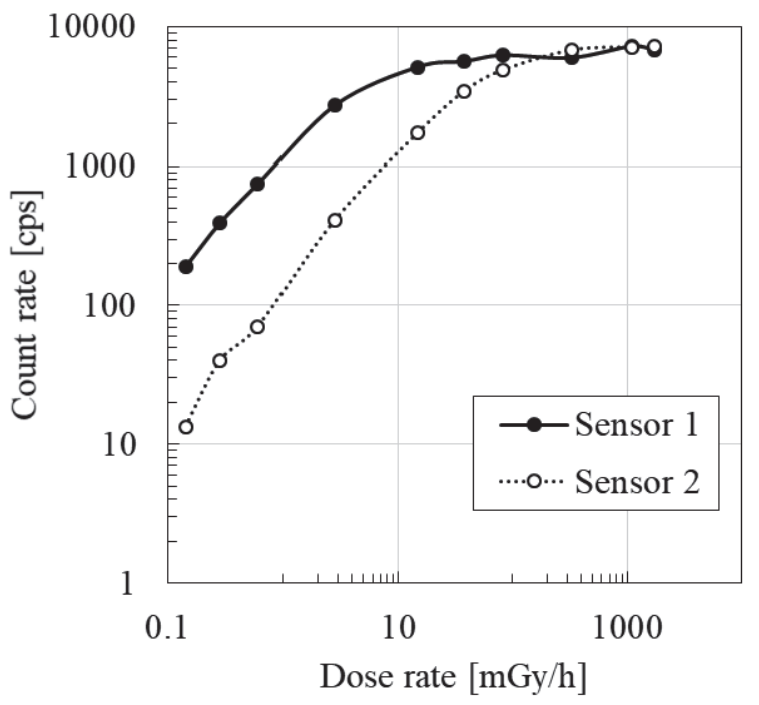

Figure 7. Count rate characteristics of the sensors.

\section{Conclusion}

We developed a wireless multisensor active personal dosimeter-tablet system. The system has great potential for energy correction using the energy spectra information. This system allows easy real-time management of the radiation exposure of medical staff, by using wireless communication. Furthermore, as the system is designed to use a tablet, high expandability can be achieved. In the future, we will evaluate the directional characteristics and the characteristics for pulse fluoroscopy for this system.

\section{References}

[1] E. Vano, J. Escaned, S. Vano-Galvan, J.M. Fernandez and C. Galvan, Importance of a patient dosimetry and clinical follow-up program in the detection of radiodermatitis after long percutaneous coronary interventions, Cardiovasc. Intervent. Radiol. 36 (2013), pp. 330-337.

[2] K. Chida, Y. Morishima, Y. Inaba, M. Taura, A. Ebata, K. Takeda, H. Shimura and M. Zuguchi, Physician received scatter radiation with angiography systems used for interventional radiology: Comparison among many $\mathrm{x}$-ray systems. Radiat. Prot. Dosimet. 149 (2012), pp. 410-416.

[3] E. Vano, L. Gonzalez, J.M. Fernandez, C. Prieto and E. Guibelalde, Influence of patient thickness and operation modes on occupational and patient radiation doses in interventional cardiology, Radiat. Prot. Dosim. 111 (2006), pp. 297-304.

[4] ICRP Statement on tissue reactions and early and late effects of radiation in normal tissues and organs - threshold doses for tissue reactions in a radiation protection context, ICRP Publication 118, Ann. ICRP 41(1/2). 2012.

[5] K. Chida, T. Takahashi, D. Ito, H. Shimura, K. Takeda and M. Zuguchi, Clarifying and visualizing sources of staff-received scattered radiation in interventional procedures. Am. J. Roentgenol. 197 (2011), pp. W900-W903.

[6] K. Chida, Y. Kaga, Y. Haga, N. Kataoka, E. Kumasaka, T. Meguro and M. Zuguchi, Occupational dose in interventional radiology procedures, Am. J. Roentgenol. 200 (2013), pp. 138-141.

[7] I. Clairand, J.M. Bordy, J. Daures, J. Debroas, M. Denozière, L. Donadille, M. Ginjaume, C. Itié, C. Koukorava, S. Krim, AL. Lebacq, P. Martin, L. Struelens, M. Sans-Mercé, M. Tosic and F. Vanhavere, Active personal dosemeters in interventional radiology: tests in laboratory conditions and in hospitals, Radiat. Prot. Dosim. 144 (2011), pp. 453-458.

[8] I. Clairand, L. Struelens, J.M. Bordy, J. Daures, J. Debroas, M. Denozières, L. Donadille, J. Gouriou, C. Itié, P. Vaz and F. d'Errico, Intercomparison of active personal dosemeters in interventional radiology, Radiat. Prot. Dosim. 129 (2008), pp. 340-345.

[9] S. Chiriotti, M. Ginjaume, E. Vano, R. Sanchez, JM. Fernandez, M.A. Duch and J. Sempau, Performance of several active personal dosemeters in interventional radiology and cardiology, Radiat. Meas. 46 (2011), pp. 1266-1270.

[10]I. Clairand, J.M. Bordy, E. Carinou, J. Daures, J. Debroas, M. Denozière, L. Donadille, M. Ginjaume, C. Itié, C. Koukoravac, S. Krim, A.L. Lebacq, P. Martin, L. Struelens, M. Sans-Merce and .F Vanhavere, Use of active personal dosemeters in interventional radiology and cardiology: Tests in laboratory conditions and recommendations - ORAMED project, Radiat. Meas. 46 (2011), pp. 1252-1257.

[11]T. Fujibuchi, H. Murazaki, T. Kuramoto, Y. Umedzu and $Y$. Ishigaki, Evaluation of an experimental production wireless dose monitoring system for radiation exposure management of medical staff, Jpn. J. Radiol. Technol. 71 (2015), pp. 691-696.

[12]Y. Inaba, K. Chida, R. Kobayashi, Y. Kaga and M. Zuguchi, Fundamental study of a real-time occupational dosimetry system for interventional radiology staff, J. Radiol. Prot. 34 (2014), pp. N65-71.

[13]Y. Inaba, K. Chida, K. Shirotori, H. Shimura, I. Yanagawa, M. Zuguchi and S. Takahashi, Comparison of the radiation dose in a cardiac IVR X-ray system, Radiat. Prot. Dosim. 143 (2011), pp. 74-80. 